\title{
Nanobiomaterials for pharmaceutical and medical applications
}

\author{
Hyukjin Lee $\cdot$ Yong-Hee Kim
}

Published online: 4 December 2013

(c) The Pharmaceutical Society of Korea 2013

Biomaterials defined as natural or synthetic materials or constructs interacting with biological systems use multidisciplinary ideas and technologies from medicine, pharmaceutics, biology, tissue engineering, material science and chemistry (Atala et al. 2010). Although biomaterials have been widely used in medical and pharmaceutical areas such as dental and bone implants, contact lenses, drug delivery, tissue regeneration, and stem cell engineering, drawbacks of classical biomaterials as well as the advent of new technology require developments of novel biomaterials and integrated systems. The development of highquality biomaterials devices or implants could be accelerated by taking the benefits of nanotechnology such as new scaffolds or nano-assemblies for tissue and stem cell engineering or targeted drug delivery (Hasirci et al. 2006). We believe healthcare can be improved by examining, visualizing, understanding and improving biological structures, processes and functions at the nano-scale level. Nanotechnologies provide a fundamental role in the development of biomaterial-based pharmaceutical and

H. Lee

College of Pharmacy, Graduate School of Pharmaceutical

Sciences, Ewha Womans University, Seoul 120-750, Korea

H. Lee

Global Top 5 Research Program, Ewha Womans University, Seoul 120-750, Korea

\section{Y.-H. Kim ( $\square)$}

Department of Bioengineering, Hanyang University,

Seoul 133-791, Korea

e-mail: yongheekim@hanyang.ac.kr

\section{Y.-H. Kim}

Institute of Bioengineering and Biopharmaceutical Research, Hanyang Univeristy, Seoul 133-791, Korea medical devices. In this way, nanobiomaterials can be building blocks for the bridge between nano and bio.

This special issue provides the current status of nanobiomaterials for pharmaceutical and medical applications. Thirteen review articles as well as three research articles fully cover a wide use of these novel materials in drug delivery system, stem cell engineering, and regenerative medicine. In the review section, first, nanoparticle systems for anti-cancer therapy and gene delivery are highlighted. Second, various polymer based biomaterials for drug delivery and molecular diagnostics are discussed in detail. Third, current stem cell engineering technology and future directions of regenerative medicine are reviewed.

The first review article from Harashima et al. brings attention to the nanoparticle systems for overcoming the multidrug resistance (MDR) of cancer cells. MDR is one of major huddles to the successful anti-cancer treatment and his review covers the details of key factors responsible for MDR in cancer cells and way to overcome the phenomena (Kibria et al. 2014). The authors provide various strategies for overcoming MDR using nanocarriers. These nanocarriers can deliver inhibitors of the MDR proteins, siRNA to silencing the target MDR protein synthesis, and modulate the uptake route of drugs to avoid MDR effect. In the second review article, Yeo et al. review the extracellular stability of nanoparticles for drug delivery applications (Liu and Yeo 2014). More specifically, the authors address the important design consideration in nanoparticulate systems to ensure the physical and functional stability of nanoparticles during circulation. An overview of stability issues observed in various nanoparticle systems is presented in this review. The third article from Kim et al. (Hwang and Kim 2014) addresses the use of nano-delivery systems, which can across the blood-brain barrier (BBB) Due to the difficulties in achieving proper delivery of a drug through the BBB, 
drug availability to the intended target in the brain is highly limited. The authors have reviewed potential strategies that can overcome the current problems for the therapy of CNS diseases.

The next article by Kim et al. (Ryu and Kim 2014) covers the therapeutic applications of bioreducible polymers as a gene delivery carrier. Bioreducible polymers have distinctive advantage over common cationic gene delivery carriers such as cytoplasm-specific release of genetic materials. The authors highlight various gene therapy approaches for the treatment of intractable diseases such as cancer, cardiovascular disease, and diabetes. In the fifth article, Lim et al. (2014) discuss about carbon-based carriers for drug delivery applications. Carbon allotropes such as graphene oxide, carbon nanotubes, and nanodiamonds (NDs) are utilized for the delivery of water insoluble drugs, antigens, antibodies, and nucleic acids to the cancer cells. The last article in nanoparticle systems describes the gold nanoparticle (AuNP) based drug delivery and molecular imaging by Lee and colleagues (Jeong et al. 2014). Surface modifications of AuNPs and their applications in delivery of small chemical drugs as well as gene materials such as pDNAs and siRNAs are discussed. Molecular probe design and imaging using AuNPs are also presented with the detailed overview of optical and fluorescence quenching properties of AuNPs.

Polymer based biomaterials and cancer stem cell diagnostics are introduced by the following reviews. Kim et al. (2014a) provides a full review of natural and synthetic polymer-based hydrogels for pharmaceutical and biomedical applications. Various hydrogel forming natural polymers including fibrin, hyaluronic acid, alginate and synthetic polymers such as poly(ethylene oxide), poly (acrylic acid), poly( $N$-isopropyl acrylamide) are well described and their medical applications are discussed. The next article by Yoo et al. review the electrospun nanofibers for drug delivery systems (Son and Yoo 2014). Electrospun nanofibers have been utilized to deliver drugs such as protein, peptide, antibodies, and small molecules. Particularly, the authors highlight the applications that utilize the combination of electrospun nanofibers with nanoparticles, microparticles, and hydrogels. Hong and colleagues (Choi and Hong 2014) introduce a controlled drug release from polymer films. Multilayer films are prepared by layer-bylayer assembly to encapsulate drugs as well as control the drug release profiles upon external stimuli. Layer forming materials include inorganic materials, nanoparticles, nanotubes, graphenes, proteins, and nucleic acid. Physical and chemical properties of these multilayer films and their versatilities are discussed. Last article in this category describes the current technologies for the in vitro diagnostics of circulating tumor cells (CTCs) from patient's blood (Kim et al. 2014b). These approaches include the label-free isolation of CTC as well as investigation of surface proteins in cancer cell lines and genomic profiling techniques for CTCs. Various fluidic channel designs and devices are also introduced for the rapid isolation of CTCs.

The last part is composed of reviews on cell reprograming, tissue engineering, and regenerative medicine. First, Egami et al. (2014) highlights the latest status of cell sheet engineering and its applications in regenerative medicine. Cell sheet engineering is a scaffold-free cell delivery system, which offers various advantages over conventional cell therapy. Using a temperature-responsive surface, a monolayer of cells can be harvested and applied in regeneration of various tissues. Especially the authors share their experiences of clinical studies using cell sheet technology and current challenge to develop a clinical product in the market. Second, Park et al. (2014) have introduced non-viral gene delivery systems for cellular reprogramming. The review discusses the use of various delivery carriers as well as cargo materials to reprogram not only the undifferentiated stem cells but also the terminally differentiated somatic cells. The authors provide recent advances in non-viral delivery approaches for reprogramming cells to pluripotency or lineage specification. At last, Hwang et al. review the controlled tissue assembly and its applications in tissue engineering (Lee 2014). The authors provide the overview of utilization magnetic nanoparticles for controlling cell functions, cell patterning, and 3D tissue assembly.

In summary, this special issue provides outstanding reviews in the field of biomaterials and nanoparticle based drug delivery system, stem cell engineering and regenerative medicine. The participants in this volume of the journal offer many of the important advances and future directions that can expand the applications in pharmaceutical and medical areas. We strongly believe this issue benefits not only the pharmaceutical and biomedical research areas, but also contributes the relevant areas such as chemistry, environment, materials, and electronics. Finally we like to thank all the authors and their collaborators who participated in organizing this special issue.

\section{References}

Atala, A., et al. 2010. Principles of regenerative medicine. San Diego: Academic Press.

Choi, D., and J. Hong. 2014. Layer-by-layer assembly of multilayer films for controlled drug release. Archives of Pharmacal Research 37: 1. doi:10.1007/s12272-013-0289-x.

Egami, M., Y. Haraguchi, T. Shimizu, M. Yamato, and T. Teruo Okano. 2014. Latest status of the clinical and industrial applications of cell sheet engineering and regenerative medicine. Archives of Pharmacal Research 37: 1. doi:10.1007/s12272-0130299-8.

Hasirci, V., et al. 2006. Nanobiomaterials: A review of the existing science and technology, and new approaches. Journal of Biomaterials Science 17(11): 1241-1268. Polymer Edition. 
Hwang, S.R., and K. Kim. 2014. Nano-enabled delivery systems across the blood-brain barrier. Archives of Pharmacal Research 37: 1 doi:10.1007/s12272-013-0272-6.

Jeong, E.H., G. Jung, C.A. Hong, and H. Lee. 2014. Gold nanoparticle (AuNP)-based drug delivery and molecular imaging for biomedical applications. Archives of Pharmacal Research 37: 1. doi:10.1007/s12272-013-0273-5.

Kibria, G., H. Hatakeyama, and H. Harashima. 2014. Cancer multidrug resistance: mechanisms involved and strategies for circumvention using a drug delivery system. Archives of Pharmacal Research 37: 1. doi:10.1007/s12272-013-0276-2.

Kim, J.K., H.J. Kim, J. Chung, J. Lee, S. Young, and Y. Kim. 2014a. Natural and synthetic biomaterials for controlled drug delivery. Archives of Pharmacal Research 37: 1. doi:10.1007/s12272-0130280-6.

Kim, K., K.H. Lee, J. Lee, and J. Choi. 2014b. Overview of current standpoints in profiling of circulating tumor cells. Archives of Pharmacal Research 37: 1. doi:10.1007/s12272-013-0285-1.

Lee, E., H. Yim, J. Heo, H. Kim, G. Jung, and N. Hwang. 2014. Application of magnetic nanoparticle for controlled tissue assembly and tissue engineering. Archives of Pharmacal Research 37: 1. doi:10.1007/s12272-013-0303-3.

Lim, D., M. Sim, L. Oh, K. Lim, and H. Park. 2014. Carbon-based drug delivery carriers for cancer therapy. Archives of Pharmacal Research 37: 1. doi:10.1007/s12272-013-0277-1.

Liu, K.C., and Y. Yeo. 2014. Extracellular stability of nanoparticulate drug carriers. Archives of Pharmacal Research 37: 1. doi:10. 1007/s12272-013-0286-0.

Park, H., J. Shin, J. Kim, and S. Cho. 2014. Nonviral delivery for reprogramming to pluripotency and differentiation. Archives of Pharmacal Research 37: 1. doi:10.1007/s12272-013-0287-z.

Ryu, K., and T. Kim. 2014. Therapeutic gene delivery using bioreducible polymers. Archives of Pharmacal Research 37: 1. doi:10.1007/s12272-013-0275-3.

Son, Y.J., and H.S. Yoo. 2014. Therapeutic applications of electrospun nanofibers for drug delivery systems. Archives of Pharmacal Research 37: 1. doi:10.1007/s12272-013-0284-2. 